\title{
磷酸二酯酶抑制剂治疗炎性肠病的研究进展
}

\author{
史建蓉 ${ }^{1}$, 马望前 ${ }^{2}$, 汤慧芳 $^{3}$ \\ 1. 浙江大学医学院附属儿童医院实验检验中心国家儿童健康与疾病临床医学研究中心 \\ 国家儿童区域医疗中心,浙江杭州 310052 \\ 2. 浙江大学医学院附属第二医院消化内科, 浙江杭州 310009 \\ 3. 浙江大学基础医学院药理学系, 浙江 杭州 310058
}

\begin{abstract}
[摘要] 炎性肠病是一种慢性复发性肠道炎性疾病, 尚无理想治疗方法。磷酸 二酯酶(PDE) 通过介导细胞内第二信使环磷酸腺苷和环磷酸鸟苷的水解影响细胞 内信号级联反应, 从而调控多种病理、生理过程。近年来, 一系列研究结果显示, PDE 抑制剂如多种PDE4 抑制剂、PDE5 抑制剂 (西地那非、他达拉非及伐地那 非)、PDE3 抑制剂(西洛他唑)、PDE9抑制剂(PF-04447943)、PDE3/PDE4 双抑制 剂 (pumafentrine) 对实验性结肠炎具有改善作用;PDE4抑制剂阿普司特在临床试验 中较替托司特的治疗优势更明显。本文就PDE抑制剂在炎性肠病治疗中的研究进 展进行综述。
\end{abstract}

[关键词] 炎性肠病; 磷酸二酯酶; 磷酸二酯酶抑制剂; 环磷酸腺苷; 环磷酸鸟苷; 综述 [中图分类号 ] R967 [ 文献标志码 ] A

\section{Research progress of phosphodiesterase inhibitors in inflam- matory bowel disease treatment}

SHI Jianrong ${ }^{1}$, MA Wangqian ${ }^{2}$, TANG Huifang ${ }^{3}$ (1. Clinical Laboratory, Children's Hospital, Zhejiang University School of Medicine, National Clinical Research Center for Child Health, National Children's Regional Medical Center, Hangzhou 310052, China; 2. Department of Gastroenterology, the Second Affiliated Hospital, Zhejiang University School of Medicine, Hangzhou 310009, China; 3. Department of Pharmacology, School of Basic Medical Sciences, Zhejiang University, Hangzhou 310058, China)

Corresponding author: TANG Huifang, E-mail: tanghuifang@zju.edu.cn, https://orcid.org/ 0000-0001-9541-1352

\begin{abstract}
[ Abstract ] Inflammatory bowel disease is a recurrent chronic intestinal inflammatory disease with unknown etiology and no effective treatment. Phosphodiesterase (PDE)
\end{abstract}

收稿日期: 2021-06-18 接受日期:2021-08-20

基金项目:国家自然科学基金(81402989); 浙江省基础公益研究计划(LGD20H010002)

第一作者:史建蓉,副主任技师,主要从事抗炎免疫药理学研究;E-mail :shijr@zju.edu.cn; https ://orcid.org/0000-0001-65830708

通信作者:汤慧芳,副教授,博士生导师,主要从事抗炎免疫药理学研究;E-mail:tanghuifang@zju.edu.cn;https://orcid.org/ 0000-0001-9541-1352 
regulates a variety of physiological and pathophysiological processes by mediating the hydrolysis of intracellular second messengers cyclic adenosine monophosphate and cyclic guanosine monophosphate. In recent years, a series of researches suggest that PDE inhibitors such as several PDE4 inhibitors, PDE5 inhibitors (sildenafil, tadalafil and vardenafil), PDE3 inhibitors (cilostazol), PDE9 inhibitor (PF-04447943) and PDE3/PDE4 double inhibitor (pumafentrine) have ameliorating effect on experimental colitis in animals. In clinical trials, PDE4 inhibitor apremilast showed more therapeutic advantage than tetomilast. This article reviews the recent research progress of PDE inhibitors in treatment of inflammatory bowel disease.

[ Key words ] Inflammatory bowel disease; Phosphodiesterase; Phosphodiesterase inhibitor; Cyclic adenosine monophosphate; Cyclic guanosine monophosphate; Review

[J Zhejiang Univ (Med Sci), 2021, 50(5): 659-665.]

[ 缩略语 ] 炎性肠病(inflammatory bowel disease,IBD); 肿瘤坏死因子(tumor necrosis factor, TNF); 磷酸二酯酶 (phosphodiesterase, PDE); 环磷酸腺苷 (cyclic adenosine monophosphate, cAMP); 环磷酸鸟苷 (cyclic guanosine monophosphate, cGMP); 葡聚糖硫酸钠 (dextran sulfate sodium, DSS);2,4,6-三硝基苯磺酸 $(2,4$, 6-trinitrobenzene sulfonic acid, TNBS); 氧化偶氮甲烷 (azoxymethane, AOM)

IBD 是一种可累及消化道所有部位的慢性特 发性炎性疾病, 以黏膜免疫紊乱、肠道炎症反复发 作为特征。溃疡性结肠炎和克罗恩病是该病的两 种表现类型, 以炎症部位和累及深度区分, 均与遗 传、免疫、生活方式和环境等因素密切相关 ${ }^{[1]}$ 。 IBD 严重影响患者生活质量, 且其发病率目前在全 球范围内呈持续上升趋势 ${ }^{[2]}$ 。然而, 目前对于IBD 的确切病因和发病机制尚未明确。一般认为,炎 症和氧化应激是 IBD 发病的关键机制 ${ }^{[3-4]}$ 。临床 上IBD 治疗药物主要包括 5-氨基水杨酸、皮质类固 醇激素、免疫抑制剂(如硫唑嘌呤)、生物制剂(如 抗 TNF- $\alpha$ 、抗整合素、抗细胞因子抗体和小分子抗 c-Jun氨基端蛋白激酶) 等 ${ }^{[5]}$ 。遗憾的是, 这些药物 仅为对症治疗而不能治愈疾病, 而且临床发现明 显的不良反应, 如贫血、肝和肾功能障碍、白细胞 减少、白内障、骨质疏松、恶性肿瘤、免疫抑制以及 机会性感染风险增加, 其中一些不良反应是不可 逆转的 ${ }^{[6]}$ 。另有研究表明, 早期手术和使用免疫 抑制剂均无法预防克罗恩病患者出现再次手术倾 向和疾病致残作用 ${ }^{[7]}$ 。因此, 寻找安全有效的 IBD 治疗药物迫在眉睫。

PDE 是细胞内第二信使 $\mathrm{cAMP}$ 和 cGMP的唯 一水解酶, 其表达水平和活性直接影响细胞内信
号级联反应,调控多种病理生理过程。根据底物 特异性、基因序列相关性、动力学特性、药理性质, PDE 分为 11 个家族 (PDE1 PDE11), 其中 PDE4、 PDE7、PDE8 选择性水解 $\mathrm{cAMP}, \mathrm{PDE} 5 、 \mathrm{PDE} 6$ 、 PDE9 特异性代谢 cGMP, 而PDE1、PDE2、PDE3、 PDE10、PDE11 同时水解 cAMP和 cGMP。目前已 发现在哺乳动物细胞中存在 21 个 $P D E$ 基因, 每个 家族都由 1 4个不同基因编码产生多个剪接变异 体 ${ }^{[8]}$ 。PDE 各家族在不同组织和细胞中比例不 同。在肠道组织中,PDE4 和PDE9 的表达量相对 较高 ${ }^{[9]}$ 。PDE 抑制剂是一类作用于靶细胞中特定 PDE 的药物, 可通过升高细胞内 cAMP和/或 cGMP 水平激活蛋白激酶 $\mathrm{A} / \mathrm{G}$, 促进下游效应蛋白磷酸 化,在不同炎症相关疾病中疗效显著 ${ }^{[10-17]}$ 。近年 来,已开展PDE 抑制剂尤其是PDE4 抑制剂作为细 胞内信号和基因转录的调节药物对 IBD 的作用机 制和治疗研究,并显示较好的抗炎作用 ${ }^{[17]}$ 。本文 就 PDE 抑制剂在 IBD 领域中的研究进展作一综 述, 以期为深人研究 IBD 发病机制及寻找治疗靶 点提供思路。

\section{PDE4抑制剂}

PDE4 是最大的,也是最早发现的PDE 家族， 
由 4 个基因 $(A 、 B 、 C 、 D)$ 编码产生的 20 25 个剪接变 异体组成。PDE4广泛分布于各种组织和细胞中, 其中 PDE4A 在人脑中表达相对较高, 在心脏和小 肠中也有表达; PDE4B 和 PDE4D 在免疫和炎症细 胞 (包括 $\mathrm{T}$ 细胞、单核细胞、巨噬细胞、中性粒细胞、 树突状细胞、嗜酸性粒细胞) 中高度表达; PDE4C 虽然存在于多数组织中, 但在肺部含量低, 在血液 系统中未见表达。在溃疡性结肠炎患者的结肠中, PDE4A、PDE4B 和PDE4D 呈现高表达 ${ }^{[18]}$ 。

过去 20 年, 已有 78 个PDE4 抑制剂进人临床 试验, 涉及哮喘、慢性阻塞性肺病、贝赫切特综合 征、银屑病、银屑病关节炎、特应性皮炎、强直性脊 柱炎、类风湿性关节炎、系统性红斑狼疮、过敏性 鼻炎、多发性硬化症、阿尔茨海默病等 ${ }^{[19]}$ 。由于呼 吸道、肠道、皮肤和关节等部位慢性炎症性疾病的 病理机制(包括一些细胞因子和免疫信号通路)相 似, 而且PDE4在炎症肠道组织中呈现高表达, 因 此 PDE4 抑制剂对 IBD 的影响及机制备受关注。 PDE4抑制剂的临床前研究提示其对实验性结肠 炎具有显著的抗炎作用,如咯利普兰(rolipram)、美 索普兰(mesopram)、罗氟司特(roflumilast)、替托司 特(tetomilast, OPC-6535)和阿普司特(apremilast) 等均能减轻DSS 诱导的 IBD 小鼠的结肠缩短程度 和组织学评分; 降低TNF- $\alpha$ 和一氧化氮水平以及髓 过氧化物酶活性、下调诱导型一氧化氮合酶基因 表达、降低肠黏膜屏障通透性及减少胶原生成等, 从而控制结肠炎进展, 减轻病理组织学改变及防 止肠道纤维化; 防止中性粒细胞迁移、中性粒细胞 与内皮细胞黏附、中性粒细胞释放超氧化物, 保护 胃肠黏膜的完整性, 并能在一定程度上预防炎症 级联反应的启动 ${ }^{[20-21]}$ 。机制研究提示, 阿普司特 可激活 cAMP介导的蛋白激酶 A-cAMP反应元件结 合蛋白和 $c A M P$ 活化交换蛋白-Ras 相关蛋白 1 通 路, 进而抑制促分裂原活化的蛋白激酶、核因子 $\kappa \mathrm{B} 、$ 磷脂酰肌醇 3 激酶-哺乳动物雷帕需素靶蛋白 (PI3K-mTOR) 和 JAK 激酶-信号转导及转录激活 因子-细胞因子信号传送阻抑物 3(JAK-STATSOCS3) 信号通路, 从而干预 PDE4 在肠道炎症中 的作用 ${ }^{[18]}$ 。

目前进入IBD临床研究的只有替托司特和阿 普司特。替托司特的临床研究始于 2003 年, 经历了 6 个 I 期、 1 个 II 期、 2 个 III 期临床试验。在 I 期 和 II 期临床试验中, 大多数受试者对替托司特的推
荐剂量 $25 \mathrm{mg} / \mathrm{d}$ 和 $50 \mathrm{mg} / \mathrm{d}$ 耐受性良好, 仅有头痛、恶 心、头晕、腹痛、呕吐等轻微不良反应, 但不会导致

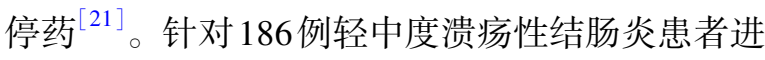
行的多中心随机双盲 II 期临床试验结果表明,与安 叞剂比较,替托司特未显著改善研究的主要终点指 标 (疾病活动指数); 对于次要终点指标数据的析因 分析表明,疾病严重程度更高的患者(如疾病活动 指数评分 7 11) 使用替托司特后疗效更好 ${ }^{[22]}$ 。 在 III期临床试验中, 替托司特与安慰剂在主要终点 指标和次要终点指标上均无显著性差异, 但替托司 特治疗组直肠出血的严重程度呈现下降趋势 ${ }^{[21]}$ 。 以上结果提示, 替托司特在高疾病活动评分的溃疡 性结肠炎患者中具有潜在的应用价值。

阿普司特于 2015 年直接进人溃疡性结肠 炎 II 期临床试验。2018 年, 在第 13 届欧洲克罗恩 病和结肠炎组织大会上, Danese 等 ${ }^{[23]}$ 公布了一项 在 170 例活动性溃疡性结肠炎患者中进行的多中 心、随机、双盲、安慰剂对照试验结果: 患者每日 2 次口服阿普司特 $30 \mathrm{mg}$ 治疗 12 周后, 根据总梅奥 评分获得临床缓解的患者比例明显大于安慰剂组 (31. 6\% 和 $13.8 \%, P<0.01)$; 梅奥内镜评分减少 1 分及以上和黏膜愈合的患者比例也较安慰剂组 显著增大。而每日 2 次口服阿普司特 $40 \mathrm{mg}$ 的患者 与安慰剂组相比, 在主要终点指标 (总梅奥评分提 示临床缓解)上无显著性差异。两种剂量组患者 超敏 C 反应蛋白和粪钙防卫蛋白的中位百分比均 有明显改善。2020年, Danese 等 ${ }^{[24]}$ 进一步报道了 后续 40 周的双盲主动治疗扩展期研究结果: 多达 $40 \%$ 的患者使用阿普司特 $30 \mathrm{mg}$ 和 $40 \mathrm{mg}$ (每日2次) 治疗后, 在观察终点即第 52 周时获得临床缓解, 且 该药具有良好的安全性和耐受性,最常见的不良反 应是头痛和恶心。阿普司特 II 期临床试验虽然初 步取得了理想结果,但其疗效仍须进一步评估和明 确。然而, 研发阿普司特的制药公司表示, 出于商 业考虑(而非缺少 II 期临床试验数据), 将不再进行 阿普司特在 IBD 患者中的 III 期临床试验 ${ }^{[25]}$ 。

由于阿普司特未显示对 PDE4 亚家族成员的 选择性，因而可能比其他PDE4抑制剂具有更大的 治疗优势 ${ }^{[26]}$, 但迄今尚未在克罗恩病患者中开展 PDE4抑制剂相关研究。

\section{PDE3 抑制剂}

$P D E 3$ 家族由 $P D E 3 A$ 和 $P D E 3 B$ 两个基因组 
成。PDE3 可同时水解 $\mathrm{cAMP}$ 和 cGMP, 其中水解 cAMP 的速率约为 cGMP 的 10 倍, 而对 $\mathrm{cGMP}$ 的亲 和力明显高于 $\mathrm{cAMP}^{[27]}$ 。PDE3A 在心脏、血管、胎 盘、海绵体平滑肌以及血小板中表达, 而 $P D E 3 B$ 在 细胞和组织中表达量更高 ${ }^{[28]}$ 。目前PDE3 抑制剂 在临床应用于心脑血管和外周血管相关疾病, 如 米力农 (milrinone) 用于难治性心力衰竭的急性期 治疗及心脏移植术前稳定和维持 ${ }^{[29]}$; 西洛他唑 (cilostazol)广泛应用于经皮冠状动脉介人治疗的 外周动脉疾病、冠状动脉疾病、脑血管疾病和间歇 性跛行等 ${ }^{[30]}$ 。

目前,PDE3 抑制剂用于IBD 治疗的临床前研 究仅两项。Rieder 等 ${ }^{[31]}$ 报道, PDE3/PDE4 双抑制 剂 pumafentrine灌胃 $11 \mathrm{~d}\left(5 \mathrm{mg} \cdot \mathrm{kg}^{-1} \cdot \mathrm{d}^{-1}\right)$ 可使 DSS 诱导结肠炎小鼠结肠组织的临床评分降低 (包 括体重、粪便稠度和出血情况等指标)、结肠长度 缩短及局部黏膜组织 TNF- $\alpha$ 产生。同时, 体外试 验结果提示, pumafentrine 能够降低豆冦酸-佛波 醇-乙酸酯 $25 \mathrm{ng} / \mathrm{mL}$ 和离子霉素 (ionomycine) $500 \mathrm{ng} / \mathrm{mL}$ 刺激DSS 小鼠脾细胞产生的 $\gamma$ 干扰素水 平, 提示 PDE3/PDE4 双抑制剂对实验性结肠炎具 有改善作用, 其机制与减少 TNF- $\alpha$ 及 $\gamma$ 干扰素有 关。Kangawa等 ${ }^{[32]}$ 报道, 西洛他唑能抑制 DSS 诱 导的小鼠结肠炎。与模型组比较, 西洛他唑 可改善腹泻症状, 防止结肠长度缩短, 降低血浆 TNF- $\alpha$ 、白介素-6、白介素-17、角质形成细胞衍生 细胞因子水平以及抑制远端结肠的稆膜损伤, 如 黏膜缺失、黏膜下水肿和炎性细胞浸润。全基因 组微阵列分析和组织学分析表明, 西洛他唑的这 些抑制作用可能涉及细胞黏附、细胞骨架调节、细 胞增殖和调亡, 与炎症细胞浸润和黏膜愈合相 关。该研究结果提示, 西洛他唑具有抗炎作用, 可 能有助于 IBD 的治疗, 但其是否具有临床应用价 值尚需进一步研究。

\section{PDE5 抑制剂}

PDE5 由单个 PDE5A基因编码, 产生三个氨基 末端变异体, 即PDE 5A1、PDE5A2和PDE 5A3。 PDE5 广泛存在于人体组织中, 但在平滑肌和血小 板中的表达相对更多。目前, PDE5 抑制剂西地那 非 (sildenafil)、他达那非 (tadalafil)、伐地那非 (vardenafil) 等已成功用于临床治疗勃起功能障碍 和肺动脉高压 ${ }^{[33]}$ 。在 IBD 研究领域, PDE5 抑制剂
的研究尚在临床前动物实验阶段。PDE5 抑制剂 对乙酸和 TNBS 诱导的实验性结肠炎以及 AOM/ DSS 诱导的结肠癌具有改善作用 ${ }^{[34-41]}$ 。

在乙酸诱导的结肠炎模型中,西地那非可降 低小鼠结肠组织中髓过氧化物酶和脂质过氧化产 物硫代巴比妥酸反应物的活性; 改善结肠黏膜组 织学变化, 其效果与泼尼松龙相似 ${ }^{[34]}$ 。这些作用 可能与西地那非增强 cGMP介导的抗氧化应激能 力有关。另有研究表明, 西地那非通过平衡氧化一 抗氧化状态、抑制活性氧代谢物的产生、减少细胞 因子如TNF- $\alpha$ 和白介素 $-1 \beta$ 的释放以及防止中性粒 细胞浸润,对乙酸诱导的大鼠结肠炎产生保护作 用 ${ }^{[38]}$ 。此外,他达那非预处理也可对乙酸诱导的 结肠损伤产生剂量依赖性的保护作用,降低结肠 组织中丙二醛水平和髓过氧化物酶活性, 提升超 氧化物歧化酶活性,降低血清白介素 $-1 \beta$ 和 TNF- $\alpha$ 水平, 其机制可能与抗炎、抗氧化和抗调亡作用 有关 ${ }^{[35]}$ 。

在 TNBS 诱导的结肠炎模型中,西地那非可通 过一氧化氮依赖机制维持氧化-抗氧化平衡、防止 细胞凋亡、超氧物产生和细胞因子释放等作用,抑 制模型大鼠结肠炎 ${ }^{[36]}$ 。Fakhfouri 等 ${ }^{[39]}$ 也报道,西 地那非可减轻TNBS诱导的大鼠结肠炎,机制涉及 cGMP和 ATP 敏感钾通道通路。另外, Margonis 等 ${ }^{[40]}$ 研究证实西地那非具有抗炎作用, 可通过降 低结肠组织 TNF- $\alpha$ 水平和改善体重而抑制 TNBS 诱导的大鼠结肠炎。联合具有抗氧化特性的药物 U-74389G, 可进一步降低组织 TNF- $\alpha$ 和丙二醛水 平、减少结肠黏膜损伤指数评分和镜下组织病理 学评分, 以及改善体重等。

除上述结肠炎动物模型外,2017年我国学者 Lin 等 ${ }^{[37]}$ 研究发现, 在 AOM/DSS 诱导的结肠炎相 关结肠癌小鼠模型中, 结肠组织PDE5 表达上调, 并且西地那非可通过抑制组织中体外髓源性抑制 细胞的浸润来调节炎症微环境,抑制 DSS 诱导的 结肠炎以及结肠炎所致结肠癌的发生。炎症是癌 变的驱动力, 因而 IBD 患者罹患结直肠癌的风险 增加 ${ }^{[42]}$ 。该研究结果为 PDE5 抑制剂在预防和治 疗 IBD 及结肠炎症相关肿瘤发生中的应用提供了 依据。同年 Islam 等 ${ }^{[41]}$ 报道, 西地那非可抑制 $\mathrm{AOM} / \mathrm{DSS}$ 小鼠的息肉形成和炎症反应,减少髓系 细胞浸润,减少诱导型一氧化氮合酶、 $\gamma$ 干扰素和 白介素-6 的表达, 从而预防 DSS 诱导的黏膜屏障 
功能障碍, 在结肠炎驱动结直肠癌的初始阶段即 发挥保护作用。以上结果提示, PDE5 抑制剂对人 类预防结肠炎及炎症所致结肠癌具有潜在应用 价值。

\section{PDE9抑制剂}

PDE9 能特异性水解 c GMP 且具有高亲和 力。目前编码基因仅发现P DE $9 A$, 该基因可产生 21 个剪接变异体。已有研究表明, PDE9A 在机体 各器官、组织中均有表达, 其在造血细胞、脑、前列 腺、结肠、小肠、脾、肾、胸腺和心脏中的表达量较 多 ${ }^{[43]}$ 。近年来, PDE9 抑制剂已广泛应用于神经 退行性变性疾病、镰状细胞病、心力衰竭和糖尿病 等相关研究。其中, PF-04447943已进人稳定性 镰状细胞病 I b 期和阿尔茨海默病 II 期临床试 验 ${ }^{[44-45]}$; BI 409306 已进人阿尔茨海默病 II 期和精

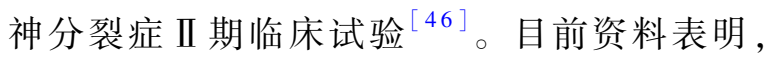
PF-04447943 和 BI 409306在患者中耐受性良好, 其中PF-04447943 具有良好的药代动力学特性, 如 分布广、吸收快 ${ }^{[44-46]}$ 。这些特点有助于进一步开 展PDE9抑制剂治疗 IBD 的相关研究。

$P D E 9 A$ 在结肠、小肠等部位呈现高表达, 与 PDE5 相似, 但PDE9对 cGMP的亲和力更强, 提示 其可能参与肠道炎症过程。有研究发现, 选择性 PDE9 抑制剂 PF-04447943 可减轻DSS 诱导的结肠 炎,其机制与激活核因子 2 , 抑制核因子 $\kappa \mathrm{B} 、$ 信号转 导及转录激活因子 3 和炎症小体活性, 抑制氧化应 激和炎症反应, 及调节调节性 $\mathrm{T}$ 细胞与辅助性 $\mathrm{T}$ 细 胞 17 平衡有关。因此,PF-04447943 可能成为一种 基于调节性 $\mathrm{T}$ 细胞与辅助性 $\mathrm{T}$ 细胞 17 比例调节黏 膜免疫来治疗 IBD 的候选药物 ${ }^{[47]}$ 。

\section{5 结 语}

在 IBD 治疗领域,特异性水解 $\mathrm{cAMP}$ 的 PDE4 抑制剂最早引起研究者的注意, 除了在实验性结 肠炎中证实多种PDE4抑制剂具有改善作用外, 目 前已完成 II 期临床试验的阿普司特体现了更好的 治疗效果及良好的安全性和耐受性。特异性水解 cGMP的PDE 抑制剂中, PDE5 抑制剂在各种诱导 性结肠炎动物模型中均表现出明显的抗炎抗氧化 作用。有研究也发现, 与 c GMP 具有强亲和力的 PDE9 抑制剂PF-04447943 能够减轻结肠炎症反 应, 改善炎症微环境, 提示 PDE9 抑制剂可能成为
治疗溃疡性结肠炎的新兴靶点药物 ${ }^{[47]}$ 。同时水 解 cAMP 和 cGMP的PDE3 抑制剂以及 PDE3/PDE4 双抑制剂在 IBD 治疗中的作用研究还处于起步 阶段。

此外,PDE7 和PDE4在许多免疫/炎症细胞中 的表达一致,并且PDE7 抑制剂避免了 PDE4 抑制 剂在临床试验中因PDE4D 亚型同时被抑制而引起 的催吐不良反应 ${ }^{[48]}$, 因而PDE4/PDE7 双抑制剂可 提供协同作用,可能比单独靶向 PDE4 具有更强的 活性,且能减少不良反应、降低PDE4 抑制剂的剂 量。PDE4/PDE7 双抑制剂可能成为包括IBD在内 的炎症和免疫疾病治疗的潜在候选药物 ${ }^{[49]}$ 。

近来,关于IBD 发病机制的研究取得了巨大 突破, 如肠类器官培养技术的发展、肠道干细胞周 围微环境调控机制的发现、对肠上皮修复的重新 认识等, 这些均有助于深人理解PDE抑制剂治疗 IBD 的作用机制及其临床应用。

利益冲突 所有作者均声明不存在利益冲突

\section{参考文献}

[1] BERNSTEIN C N. Review article: changes in the epidemiology of inflammatory bowel disease-clues for aetiology [J]. Aliment Pharmacol Ther, 2017, 46(10): 911-919.

[2] NG S C, SHI H Y, HAMIDI N, et al. Worldwide incidence and prevalence of inflammatory bowel disease in the 21st century: a systematic review of populationbased studies[J]. Lancet, 2017, 390(10114): 2769-2778.

[3] BOURGONJE A R, FEELISCH M, FABER K N, et al. Oxidative stress and redox-modulating therapeutics in inflammatory bowel disease $[\mathrm{J}]$. Trends Mol Med, 2020, 26(11): 1034-1046

[4] TIAN T, WANG Z, ZHANG J. Pathomechanisms of oxidative stress in inflammatory bowel disease and potential antioxidant therapies $[\mathrm{J}]$. Oxid Med Cell Longev, 2017, 2017: 1-18.

[5] RUBIN D T, ANANTHAKRISHNAN A N, SIEGEL C A, et al. ACG clinical guideline: ulcerative colitis in adults $[J]$. Am J Gastroenterol, 2019, 114(3): 384-413.

[6] HINDRYCKX P, NOVAK G, COSTANZO A, et al. Disease-related and drug-induced skin manifestations in inflammatory bowel disease $[\mathrm{J}]$. Expert Rev Gastroenterol Hepatol, 2017, 11(3): 203-214.

[7] TRIPATHI K, FEUERSTEIN J D. New developments in ulcerative colitis: latest evidence on management, treatment, and maintenance $[\mathrm{J}]$. Drugs Context, 
2019, 8: 212572.

[8] AHMAD F, MURATA T, SHIMIZU K, et al. Cyclic nucleotide phosphodiesterases: important signaling modulators and therapeutic targets $[\mathrm{J}]$. Oral Dis, 2015, 21(1): 25-50

[9] SALARI P, ABDOLLAHI M. Phosphodiesterase inhibitors in inflammatory bowel disease $[\mathrm{J}]$. Expert Opin Investig Drugs, 2012, 21(3): 261-264.

[10] MOKRA D, MOKRY J. Phosphodiesterase inhibitors in acute lung injury: what are the perspectives? $[\mathrm{J}]$. Int J Mol Sci, 2021, 22(4): 1929.

[11] GIORGI M, CARDARELLI S, RAGUSA F, et al. Phosphodiesterase inhibitors: could they be beneficial for the treatment of COVID-19?[J]. Int J Mol Sci, 2020, 21(15): 5338.

[12] YASMEEN S, AKRAM B H, HAINSWORTH A H, et al. Cyclic nucleotide phosphodiesterases (PDEs) and endothelial function in ischaemic stroke. A review $[\mathrm{J}]$. Cell Signal, 2019, 61: 108-119.

[13] SCHEPERS M, TIANE A, PAES D, et al. Targeting phosphodiesterases-towards a tailor-made approach in multiple sclerosis treatment $[\mathrm{J}]$. Front Immunol, 2019, 10: 1727

[14] WAHLANG B, MCCLAIN C, BARVE S, et al. Role of cAMP and phosphodiesterase signaling in liver health and disease $[\mathrm{J}]$. Cell Signal, 2018, 49: 105-115.

[15] PENG T, GONG J, JIN Y, et al. Inhibitors of phosphodiesterase as cancer therapeutics $[\mathrm{J}]$. Eur $\mathbf{J}$ Medicinal Chem, 2018, 150: 742-756.

[16] KNOTT E P, ASSI M, RAO S N R, et al. Phosphodiesterase inhibitors as a therapeutic approach to neuroprotection and repair[J]. Int J Mol Sci, 2017, 18(4): 696.

[17] SCHMidT C, GRUNERT P C, STALLMACH A. An update for pharmacologists on new treatment options for inflammatory bowel disease: the clinicians' perspective[J]. Front Pharmacol, 2021, 12: 655054.

[18] LI H, FAN C, FENG C, et al. Inhibition of phosphodiesterase-4 attenuates murine ulcerative colitis through interference with mucosal immunity $[\mathrm{J}]$. Br J Pharmacol, 2019, 176(7): bph.14667.

[19] LI H, ZUO J, TANG W. Phosphodiesterase-4 inhibitors for the treatment of inflammatory diseases $[\mathrm{J}]$. Front Pharmacol, 2018, 9: 1048.

[20］薛恩复, 马望前, 汤慧芳. 磷酸二酯酶4抑制剂治疗 炎症性肠病研究进展 $[\mathrm{J}]$. 中国药理学与毒理学杂 志, 2019, 33(7): 535-541.

XUE Enfu, MA Wangqian, TANG Huifang. Research progress in phosphodiesterase 4 inhibitors in treatment of inflammatory bowel diseases $[\mathrm{J}]$. Chinese Journal of Pharmacology and Toxicology, 2019, 33(7): 535541. (in Chinese)

[21] KESHAVARZIAN A, MUTLU E, GUZMAN J P, et al.
Phosphodiesterase 4 inhibitors and inflammatory bowel disease: emerging therapies in inflammatory bowel disease $[\mathrm{J}]$. Expert Opin Investig Drugs, 2007, 16(9): 1489-1506.

[22] SCHREIBER S, KESHAVARZIAN A, ISAACS K L, et al. A randomized, placebo-controlled, phase II study of tetomilast in active ulcerative colitis $[\mathrm{J}]$. Gastroenterology, 2007, 132(1): 76-86.

[23] DANESE S, NEURATH M, KOPON A, et al. Apremilast for active ulcerative colitis: a phase 2, randomised, double-blind, placebo-controlled induction study $[\mathrm{J}]$. Gastroenterology, 2018, 154(6): s-167.

[24] DANESE S, NEURATH M F, KOPO A, et al. Effects of apremilast, an oral inhibitor of phosphodiesterase 4, in a randomized trial of patients with active ulcerative colitis $[\mathrm{J} / \mathrm{OL}]$. Clin Gastroenterol Hepatol, 2020, 18(11): 2526-2534.e9.

[25] MISSELWITZ B, JUILLERAT P, SULZ M C, et al. Emerging treatment options in inflammatory bowel disease: Janus kinases, stem cells, and more $[\mathrm{J}]$. Digestion, 2020, 101(Suppl. 1): 69-82.

[26] SPADACCINI M, D'ALESSIO S, PEYRIN-BIROULET L, et al. PDE4 inhibition and inflammatory bowel disease: a novel therapeutic avenue[J]. Int J Mol Sci, 2017, 18(6): 1276.

[27] AHMAD F, DEGERMAN E, MANGANIELLO V C. Cyclic nucleotide phosphodiesterase 3 signaling complexes $[\mathrm{J}]$. Horm Metab Res, 2012, 44(10): 776-785.

[28] BEGUM N, SHEN W, MANGANIELLO V. Role of PDE3A in regulation of cell cycle progression in mouse vascular smooth muscle cells and oocytes: implications in cardiovascular diseases and infertility $[\mathrm{J}]$. Curr Opin Pharmacol, 2011, 11(6): 725-729.

[29] AZEVEDO M F, FAUCZ F R, BIMPAKI E, et al. Clinical and molecular genetics of the phosphodiesterases (PDEs) $[\mathrm{J}]$. Endocrine Rev, 2014, 35(2): 195-233.

[30] ZHENG H, YANG H, GONG D, et al. Progress in the mechanism and clinical application of cilostazol $[\mathrm{J}]$. Curr Top Med Chem, 2020, 19(31): 2919-2936.

[31 RIEDER F, SIEGMUND B, BUNDSCHUH D S, et al. The selective phosphodiesterase 4 inhibitor roflumilast and phosphodiesterase 3/4 inhibitor pumafentrine reduce clinical score and TNF expression in experimental colitis in mice[J/OL]. PLoS One, 2013, 8(2): e56867.

[32] KANGAWA Y, YOSHIDA T, ABE H, et al. Antiinflammatory effects of the selective phosphodiesterase 3 inhibitor, cilostazol, and antioxidants, enzymaticallymodified isoquercitrin and $\alpha$-lipoic acid, reduce dextran sulphate sodium-induced colorectal mucosal injury in mice[J]. Exp Toxicologic Pathol, 2017, 
69(4): 179-186.

[33] ALA M, MOHAMMAD JAFARI R, DEHPOUR A R. Sildenafil beyond erectile dysfunction and pulmonary arterial hypertension: thinking about new indications $[\mathrm{J}]$. Fundam Clin Pharmacol, 2021, 35(2): 235-259.

[34] KHOSHAKHLAGH P, BAHROLOLOUMI-SHAPOURABADI M, MOHAMMADIRAD A, et al. Beneficial effect of phosphodiesterase-5 inhibitor in experimental inflammatory bowel disease; molecular evidence for involvement of oxidative stress[J]. Toxicol Mech Methods, 2007, 17(5): 281-288.

[35] DINA A A L, WALAA Y A, OLFAT G S, et al. Evaluation of the colo-protective effects of tadalafil in an experimental model of ulcerative colitis in rats $[\mathrm{J}]$. Afr J Pharm Pharmacol, 2017, 11(32): 385-393.

[36] KARAKOYUN B, USLU U, ERCAN F, et al. The effect of phosphodiesterase-5 inhibition by sildenafil citrate on inflammation and apoptosis in rat experimental colitis[J]. Life Sci, 2011, 89(11-12): 402-407.

[37] LIN S, WANG J, WANG L, et al. Phosphodiesterase-5 inhibition suppresses colonic inflammation-induced tumorigenesis via blocking the recruitment of MDSC [J]. Am J Cancer Res, 2017, 7(1): 41-52.

[38 ] ISERI S O, ERSOY Y, ERCAN F, et al. The effect of sildenafil, a phosphodiesterase-5 inhibitor, on acetic acid-induced colonic inflammation in the $\operatorname{rat}[\mathbf{J}]$. J Gastroenterol Hepatol, 2009, 24(6): 1142-1148.

[39] FAKHFOURI G, RAHIMIAN R, HASHEMI S, et al. Sildenafil attenuates TNBS-induced colitis in rats: possible involvement of cGMP and KATP channels $[\mathrm{J}]$. Fundamental Clin Pharmacol, 2012, 26(2): 190-193.

[40] MARGONIS G A, CHRISTOLOUKAS N, ANTONIOU E, et al. Effectiveness of sildenafil and U-74389G in a rat model of colitis $[J]$. J Surg Res, 2015, 193(2): 667-674.

[41] ISLAM B N, SHARMAN S K, HOU Y, et al. Sildenafil suppresses inflammation-driven colorectal cancer in mice$[$ J ]. Cancer Prev Res, 2017, 10(7): 377-388.
[42] BEAUGERIE L, ITZKOWITZ S H. Cancers complicating inflammatory bowel disease[J]. N Engl J Med, 2015, 372(15): 1441-1452.

[43] CASTRO A, JEREZ M J, GIL C, et al. Cyclic nucleotide phosphodiesterases and their role in immunomodulatory responses: advances in the development of specific phosphodiesterase inhibitors $[\mathrm{J}]$. Med Res Rev, 2005, 25(2): 229-244.

[44] CHARNIGO R J, BEIDLER D, RYBIN D, et al. PF 04447943, a phosphodiesterase 9A inhibitor, in stable sickle cell disease patients: a phase I b randomized, placebo-controlled study $[J]$. Clin Transl Sci, 2019, 12(2): 180-188

[45] SCHWAM E M, NICHOLAS T, CHEW R, et al. A multicenter, double-blind, placebo-controlled trial of the PDE9A inhibitor, PF-04447943, in Alzheimer's disease $[\mathrm{J}]$. Curr Alzheimer Res, 2014, 11(5): 413-421.

[46] PRICKAERTS J, HECKMAN P R A, BLOKLAND A. Investigational phosphodiesterase inhibitors in phase I and phase II clinical trials for Alzheimer's disease $[\mathrm{J}]$. Expert Opin Investig Drugs, 2017, 26(9): 1033-1048.

[47] RANA M N, LU J, XUE E, et al. PDE9 inhibitor PF04447943 attenuates DSS-induced colitis by suppressing oxidative stress, inflammation, and regulating Tcell polarization $[\mathrm{J}]$. Front Pharmacol, 2021, 12: 643215.

[48] GARCÍA A M, BREA J, MORALES-GARCÍA J A, et al. Modulation of camp-specific PDE without emetogenic activity: new sulfide-like PDE7 inhibitors[J]. J Med Chem, 2014, 57(20): 8590-8607.

[49] RUILOVÁ V, WIERCZEK A, VANDA D, et al. New imidazopyridines with phosphodiesterase 4 and 7 inhibitory activity and their efficacy in animal models of inflammatory and autoimmune diseases $[\mathbf{J}]$. Eur $\mathbf{J}$ Medicinal Chem, 2021, 209: 112854.

$$
\text { [本文编辑 沈 敏 余 方] }
$$

